\title{
Cardiovascular Risk Prediction in Korean Adults
}

\author{
Young Gyu Cho \\ Department of Family Medicine, Inje University Seoul Paik Hospital, Inje University College of Medicine, Seoul, Korea
}

Cardiovascular risk prediction is a key element in identifying high-risk individuals, for planning preventive interventions. ${ }^{1)}$ Numerous equations have been developed and validated to estimate cardiovascular risk. The Framingham Risk Score (FRS) is the first equation for risk prediction, which was adopted by the Adult Treatment Panel III and has been widely used worldwide. Since the FRS was developed from the Framingham cohort, an exclusively white US population, it overestimated cardiovascular risk in cohorts with low background risk of cardiovascular disease (CVD), for example, cohorts in Europe and Asia. ${ }^{2}$ In 2013, the American College of Cardiology (ACC) and the American Heart Association (AHA) reported the Pooled Cohort Equations (PCE) to estimate the 10-year risk of atherosclerotic cardiovascular disease (ASCVD) for non-Hispanic African-Americans and non-Hispanic whites aged 40-79 years. They recommended that PCE for non-Hispanic whites may be considered for risk estimation in populations other than non-Hispanic African Americans and nonHispanic whites, although the risk may be overestimated for Hispanic and Asian population. ${ }^{3)}$

Since the release of the 2013 ACC/AHA guidelines, it has been argued whether the new guidelines and PCE are applicable for Asian population including Koreans. The Japan Atherosclerosis Society commented that it is not necessary to change their guidelines because applying PCE to Asian population can overestimate the risk. ${ }^{4)}$ The committee formulating the Korean guidelines for management of dyslipidemia mentioned that it is not appropriate to quote the risk equations developed in Western population as mortality from coronary heart disease in Korea is among the lowest worldwide. ${ }^{5)}$ They suggested that it is absolutely needed to develop and validate a tool to estimate cardiovascular risk in Koreans. Jung et al. ${ }^{6}$ ) examined the performance of PCE in the Korean Heart Study
(KHS) cohort and developed a Korean Risk Prediction Model (KRPM) for ASCVD. In their study, PCE for non-Hispanic whites overestimated the 10 -year ASCVD risk by $56.5 \%$ in men but underestimated the risk by $27.9 \%$ in women. Contrastingly, KRPM provided an accurate prediction of actual ASCVD events in the KHS cohort. They concluded that PCE should not be directly applied for cardiovascular risk prediction in Korean population. KRPM should be further validated in another cohort before it is adopted as the ASCVD risk calculator by the Korean guidelines.

In the present issue, Kim et al. ${ }^{7)}$ examined the relationship between the serum 25-hydroxyvitamin D level and the ASCVD risk score calculated by PCE in Korean adults, using the data from the Korea National Health and Nutrition Examination Survey, 2014. The study subjects consisted of 1,134 Koreans (564 men and 570 women) aged $40-79$ years. They presented an unexpected finding that serum 25-hydroxyvitamin D level was positively related with ASCVD risk score in women. Previous studies have reported that vitamin D deficiency is associated with the risk of CVD. ${ }^{8)}$ Age and vitamin D supplement intake might have acted as confounding factors in their study. Many healthy, elderly individuals pass the 7.5\% ASCVD risk threshold estimated by PCE due to chronologic age alone. ${ }^{9)}$ In addition, serum 25-hydroxyvitamin D level positively correlates with age. ${ }^{10)}$ Therefore, it seems unlikely that women with high level of serum 25-hydroxyvitamin D have increased cardiovascular risk, despite a positive relationship between serum 25-hydroxyvitamin D level and ASCVD risk score by PCE in women, as shown in their study.

The Korean guidelines do not recommend PCE to estimate 10-year risk of ASCVD. Applying PCE can result in overestimation of the ASCVD risk in Korean population and lead to wrong conclusions in Korean studies. It is anticipated that 
KRPM will be endorsed as the ASCVD risk calculator by the Korean guidelines after further research to validate its utility.

\section{CONFLICT OF INTEREST}

No potential conflict of interest relevant to this article is reported.

\section{REFERENCES}

1. Lloyd-Jones DM. Cardiovascular risk prediction: basic concepts, current status, and future directions. Circulation 2010;121:1768-77.

2. Cooney MT, Dudina AL, Graham IM. Value and limitations of existing scores for the assessment of cardiovascular risk: a review for clinicians. J Am Coll Cardiol 2009;54:1209-27.

3. Goff DC Jr, Lloyd-Jones DM, Bennett G, Coady S, D'Agostino RB, Gibbons R, et al. 2013 ACC/AHA guideline on the assessment of cardiovascular risk: a report of the American College of Cardiology/American Heart Association Task Force on practice guidelines. Circulation 2014;129(25 Suppl 2):S49-73.

4. Arai H, Sasaki J, Teramoto T. Comment on the new guidelines in USA by the JAS guidelines committee. J Atheroscler Thromb 2014;21:79-81.

5. Committee for Guidelines for Management of Dyslipidemia. 2015 Ko- rean Guidelines for Management of Dyslipidemia. J Lipid Atheroscler 2015;4:61-92.

6. Jung KJ, Jang Y, Oh DJ, Oh BH, Lee SH, Park SW, et al. The ACC/AHA 2013 pooled cohort equations compared to a Korean Risk Prediction Model for atherosclerotic cardiovascular disease. Atherosclerosis 2015;242:367-75.

7. Kim MH, Kim YS, Oh HJ, Kwon YR, Kim HW. The association between 10-year atherosclerotic cardiovascular diseases risk score calculated using 2013 American College of Cardiology/American Heart Association guidelines and serum 25-hydroxyvitamin D level among aged 4079 years in Korea: the sixth Korea National Health and Nutrition Examination Surveys. Korean J Fam Med 2018;39:174-9.

8. Judd SE, Tangpricha V. Vitamin D deficiency and risk for cardiovascular disease. Am J Med Sci 2009;338:40-4.

9. Amin NP, Martin SS, Blaha MJ, Nasir K, Blumenthal RS, Michos ED. Headed in the right direction but at risk for miscalculation: a critical appraisal of the 2013 ACC/AHA risk assessment guidelines. J Am Coll Cardiol 2014;63(25 Pt A):2789-94.

10. Kim MY, Kim MJ, Ly SY. Vitamin D intake, serum 25OHD, and bone mineral density of Korean adults: based on the Korea National Health and Nutrition Examination Survey (KNHANES, 2011). J Nutr Health 2016;49:437-46. 